\title{
An Efficient Approach for Data Transmission in RFID Middleware
}

\author{
Hongying LIU1, Satoshi GOTO² and Junhuai $\mathrm{LI}^{3}$ \\ 1,2Graduate School of Information, Production and Systems, Waseda University, \\ ${ }^{3}$ Computer Science \& Engineering School, Xi'An University of Technology, \\ 1,2Japan \\ ${ }^{3}$ China
}

\section{Introduction}

RFID projects have been deployed in many enterprises profoundly. From the perspective of architecture, a RFID application system includes three parts: hardware, software and middleware. The hardware consists of RFID readers, tags and antennas. Software refers to the enterprise management information system which has been operated for a long time. Middleware is the most time-consuming part. How to import data from RFID reader to management information system? Who is responsible for this task? Whether or not adopt off-the-shelf RFID components? All the above issues should be carefully considered.

RFID middleware is system software that collects a large volume of raw data from heterogeneous RFID devices, filters them and summarizes them into meaningful information, delivers this information to applications and makes them interoperate with legacy systems. The implementation of RFID middleware in enterprises can provide flexible configuration. Parameters of related components can be set. And then only the required information is imported to the system. When extra RFID readers are added to the system, there is no need to develop program again. The RFID middleware will take care of all these changes.

However, the performance of RFID middleware is not satisfactory. In particular, when Web Service is used to bridge the difference of diversified platforms, the efficiency of data transmission is decreased. A Web Service is a software system identified by a URI, whose public interfaces and bindings are defined and described using XML. Web Services allow access to software components through standard web technologies, regardless of platforms, implementation languages, etc. This technology has impacted RFID-based applications in a profound way. For the mobile client system, Web Services enable the integration of various applications in a distributed environment from the resource-limited terminals. Though its performance optimization poses challenges for researchers.

Several methods and strategies have been proposed to improve it. In our paper, we design a Context-Store and apply it to the exchange process of SOAP messages between mobile terminal and server. A SOAP message which includes three sections: SOAP Envelop, SOAP Header and SOAP Body, are separated into 2 groups, one is the static part, and the other is the dynamic part. The main idea of our approach is to store the static part while only transmit the dynamic part through network. Then on the receiver side, we can assemble and obtain the whole message.

Source: Radio Frequency Identification Fundamentals and Applications, Bringing Research to Practice, Book edited by: Cristina Turcu, ISBN 978-953-7619-73-2, pp. 278, February 2010, INTECH, Croatia, downloaded from SCIYO.COM 
Under the experiment of a mobile client server system, RFID data is transmitted between the J2ME platform on PDA and the Microsoft Windows Server 2003 operating system on server. SOAP messages in different lengths are tested. Compared with the general method and compressing method, our Context-Store approach has the least average response time. It is efficient. Finally, the prototype system, which is implemented and applied to production lines in a manufactory, is described in details. Processing data collected from RFID tags attached to the product on production line is packed into SOAP messages and sent to application server by Wireless LAN. After confirmation, the PDA client can access application server to update processing data continuously.

\section{Background}

Radio Frequency Identification (RFID) is a kind of Auto-Recognition technology that allows RFID reader to read data from RFID tags via radio signals from a distance and without line of sight. With significant technology advantages over bar code identification systems, RFID has been gradually adopted and deployed in a wide range of applications, such as access control, item tracking, highway tolls, production line auto-management, supply chain management and so on. A typical RFID system consists of tags (transponders), readers, antennas embedded both in tags and readers.

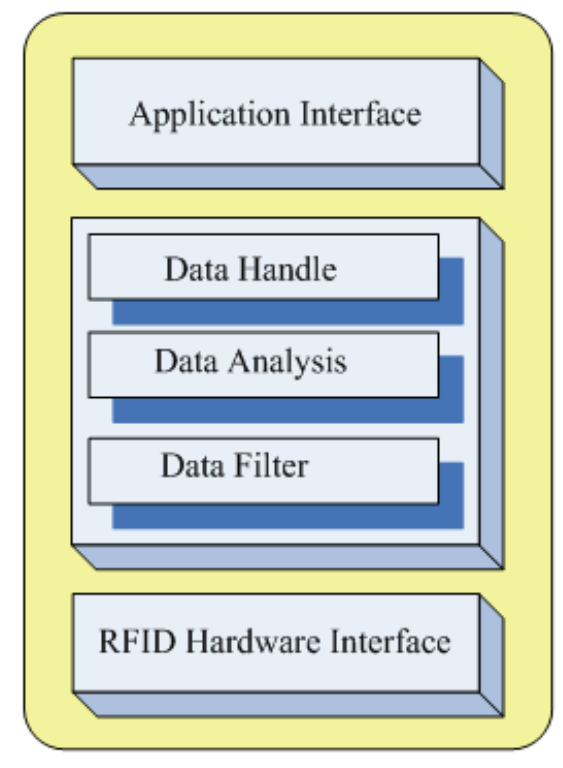

Fig. 1. Typical structure of RFID middleware

RFID middleware is system software that collects a large volume of raw data from heterogeneous RFID devices, filters them and summarizes them into meaningful information, delivers this information to applications and makes them interoperate with legacy systems (Taesu Cheong \& Youngil Kim, 2005). Therefore the basic functions of RFID middleware are supporting the independency of the multi-protocol of heterogeneous readers and playing the role of a broker for data exchange. 
A typical RFID middleware has such abstract structure as shown in Fig. 1. The Hardware Interface directly communicates with RFID readers and other terminal devices. The middle layer is responsible for data management and data transmission, such as filtering data, analyzing data and handle data. The Application Interface provides friendly access interface for upper layer applications including various enterprise and its partners. For instance, we can invoke Web Services in this Interface to accomplish broader applications. A number of works on RFID middleware has been published (Christian Floerkemeier \& Matthias Lampe, 2005; Young-Il Kim et al., 2006).

A Web Service is a software system identified by a URI(Universal Resource Identifier), whose public interfaces and bindings are defined and described using XML (World Wide Web Consortium, 2008). Web Services allow access to software components through standard Web technologies, regardless of platforms, implementation languages, etc. This technology has impacted RFID-based applications in a profound way. Especially for the mobile client system, Web Services enable the integration of diversifying applications in a distributed environment from the resource-limited terminals. It transmits business data on Web by virtue of HTTP (Hypertext Transfer Protocol, a universal protocol used on Web) and SOAP (Simple Object Access Protocol). The transmission process is illustrated in Fig.2. The client creates a SOAP message embedded in a HTTP POST (a request method used in HTTP) request according to the WSDL (Web Services Description Language), and then sends to server through network. The Web Service request processor on the server will deal with this message. It parses the SOAP message, invokes corresponding Web Services, and then creates response message. The server sends back this SOAP response message to client by HTTP.

SOAP (World Wide Web Consortium, 2007) is a lightweight and simple protocol which is based on XML. It is designed to transmit structured and solidified information, and can be used together with many protocols and formats on the Internet, such as HTTP, SMTP (Simple Message Transfer Protocol) and MIME protocol (Multipurpose Internet Mail Extension) etc.

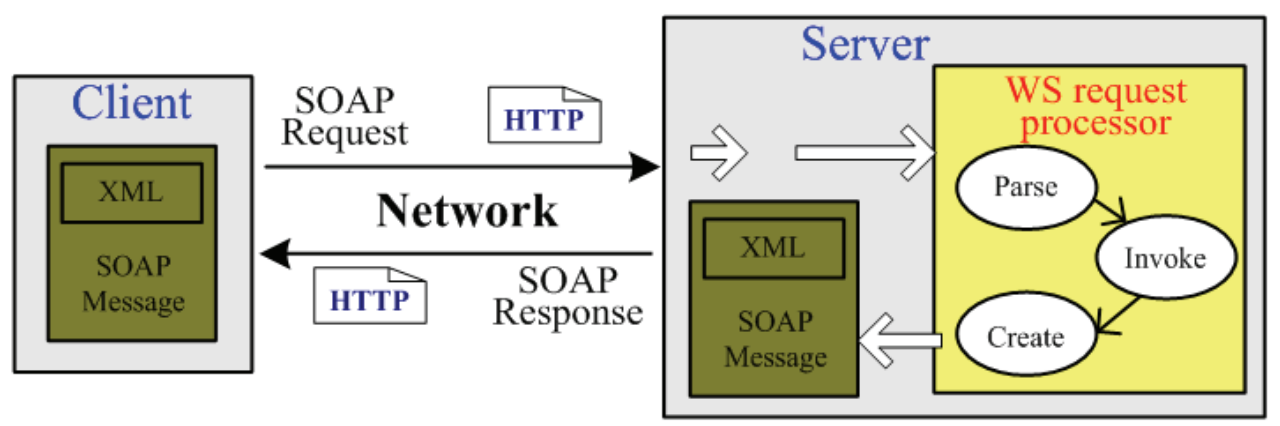

Fig. 2. Data transmission process with Web Services

For the application of Web Services-based RFID either within an enterprise or between different enterprises, several meaningful attempts have been done. Generally, when the technique of Web Services is applied to RFID system within an enterprise, the main purpose is to deal with the information in enterprise network, and make advantage of the processing capability of RFID middleware system. Clemens Kerer et al (Clemens Kerer et al., 2004) design a so-called "Presence Manager Service" to manage the list of people attending the conference. This application tries to combine Web Services with RFID devices and send the 
data collected from RFID reader to organizer of the conference. It is a unique application of which functions are not complex and the data involved is in a small scale. So it is not appropriate to apply this method to large scale systems, such as supply chains in logistics. When Web Services is used to assist the information exchange between different enterprises, its characteristic of supporting real-time data share and mutual interchange of $B$ to $B$ is revealed. Both the information on RFID tags and the processing status are provided to users. Niranjan and Aura Ganz (Niranjan \& Aura Ganz, 2004) propose a framework for a multimedia information transmission system in which diverse wireless networks and devices communicate. The client components which include lap-top, PDAs, are connected to smart objects through Web Services in dynamic environment. Each network provides its own resources to other networks by Web Services. B S Prabhu et al (B S Prabhu et al., 2006) introduce a framework for RFID middle ware. The task of this middle ware is to serve the information exchange between enterprises. Each enterprise communicates with others through Web Services across the boundaries.

Though these Web Services-based performance improvement poses challenges for researchers. There are some achievements both from industrial and academic institutes. V Prabakar et al. focused on the manageability of Web services in RFID application (V Prabakar et al., 2006; Han Chen et al., 2005). K.Devaram and D.Andresen suggested to cache SOAP messages in client side to enhance the performance (K Devaram \& D Andresen, 2003) Seshasayee B and Noah Mendelsohn et al encoded the SOAP message by binary codes (Noah Mendelsohn et al., 2004; Seshasayee B et al., 2004). Mike Nikitas compressed SOAP messages (Mike Nikitas, 2003). However the above methods cause some negative effects. The binary encoding loses the advantage of readability of the XML-based SOAP messages. The compressing method produces extra compressing and decompressing time costs and loses the access transparency.

In this paper, we design and realize a Context-Store approach to improve the Web Services performance in RFID middleware. Both the experiments and the application example show its efficiency. The remainder of this paper is organized as follows. Section 3 presents our framework. Section 4 describes the realization of our approach in detail. Section 5 shows the experiments. Section 6 outlines the application in manufactory. Section 7 draws conclusions and suggests future research.

\section{Framework design}

According to the research work (Julio Fernandez et al., 2005; Toyotaro Suzumura et al., 2005), the major factor affects the performance of Web Services is the cost of SOAP message transmission. So the purpose of our approach is to reduce the message length transmitted in network. The main idea is to store the static part while only transmit the dynamic part through network. Then on the receiver side, we can assemble and obtain the whole message. SOAP message (World Wide Web Consortium, 2007) is a sort of XML document. It comprises 3 sections: SOAP Envelop, SOAP Header and SOAP Body. In the following discussion, we divide it into 2 parts, the static part which contains the SOAP Envelop, fixed SOAP Header (assessing Web Services not for the first time) and Tag information such as "<? xml version="1.0" enconding="utf-8" ?>", and the dynamic part which contains SOAP Body and dynamic SOAP Header (accessing Web Services for the first time).

The framework of the system is shown in Fig. 3. This framework includes mobile terminals which are capable of collecting data from RFID tags, Context-Store, Web Services server and transmission channels such as network and so on. The mobile terminal sends requests by SOAP messages. The Web Services server responses. Context-Store is a kind of file storage 


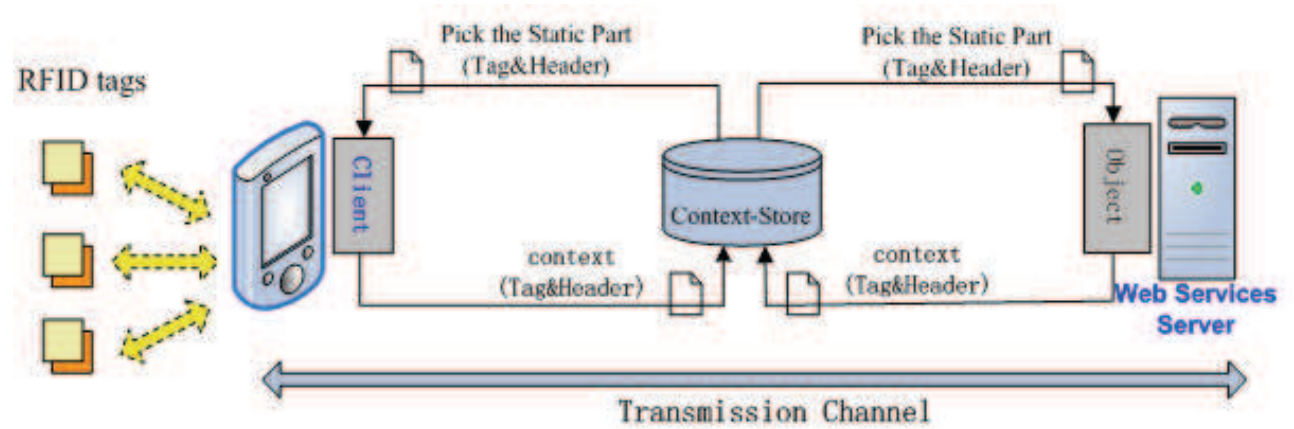

Fig. 3. System framework

structure, which can be accessed by client and server. It stores the context information such as SOAP Envelop, fixed SOAP Header and tag information. To choose a suitable structure as Context-Store is up to the specific application environment. For example, considering the limited resources on mobile terminal, we adopt a simple text file structure. The static part of SOAP message stores in Context-Store, while the dynamic part is transmitted through network. Then after assemble, we can obtain the whole message from the mobile client side or the Web Services server side.

\section{Approach description}

\subsection{Context-Store construction}

The main idea of our approach is to reduce the lengths of SOAP messages transmitted through network to enhance the performance of Web Services.

$$
\begin{aligned}
& \text { e?xml version=" } 1.0 \text { " encoding="utf-8"? }>+ \\
& \text { ¿soap:Envelope } \\
& \text { xmlns:soap="http:ilschemas } \text {.xmlsoap.org/soaplervelope/" } \\
& \text { xmlns: } x s i=" \text { http://woww.w3 .org/2001/XMLSchema-instance" } \\
& \text { xmlns: } x s d=" h t t p: / / \text { wow: } w 3 \text {.org/2001/XMLSchema" }> \\
& \Leftrightarrow \text { soap:Header }>\text { MyHeader }
\end{aligned}
$$

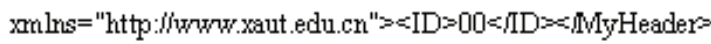

$$
\begin{aligned}
& \text { sisoap:Header }=4
\end{aligned}
$$

Fig. 4. A Context-Store template

The mobile terminal communicates with the Context-Store in the following steps:

Step 1. Create a context with ID;

Step 2. Store a specific context matched to the ID;

Step 3. Pick the context from Context-Store;

The server communicates with the Context-Store in the following steps:

Step 1. Store the context information which access the Web Services for the first time;

Step 2. Pick the context from Context-Store;

We construct a template for the Context-Store in Fig. 4 to initiate communication process between mobile client and server in which we set the ID as "00". Because of the fast speed of assess ordinary text file, in our realization, we construct TXT files as Context-Store to store 
context information in both mobile client and server side. They are symmetrically nominated as Client_Context_Store and Server_Context_Store respectively.

\subsection{SOAP expansion on mobile client}

On the mobile terminal client side, we expand the communication process by the following algorithm, and it is illustrated in Fig. 5.

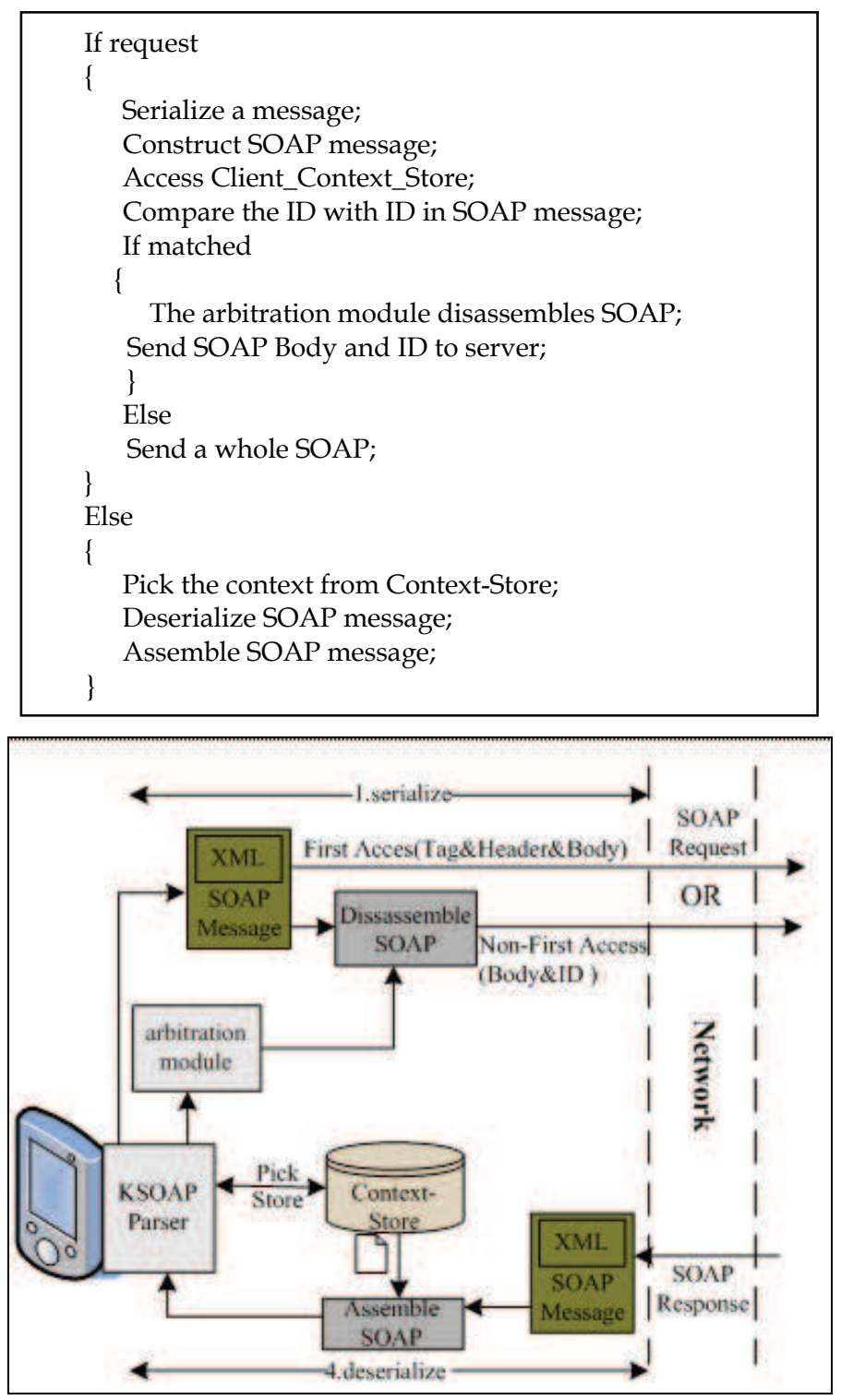

Fig. 5. Algorithm on mobile client 


\subsection{SOAP expansion on Web Services server}

On the Web Services server side, we expand the communication process by the following algorithm, and it is illustrated in Fig. 6 .
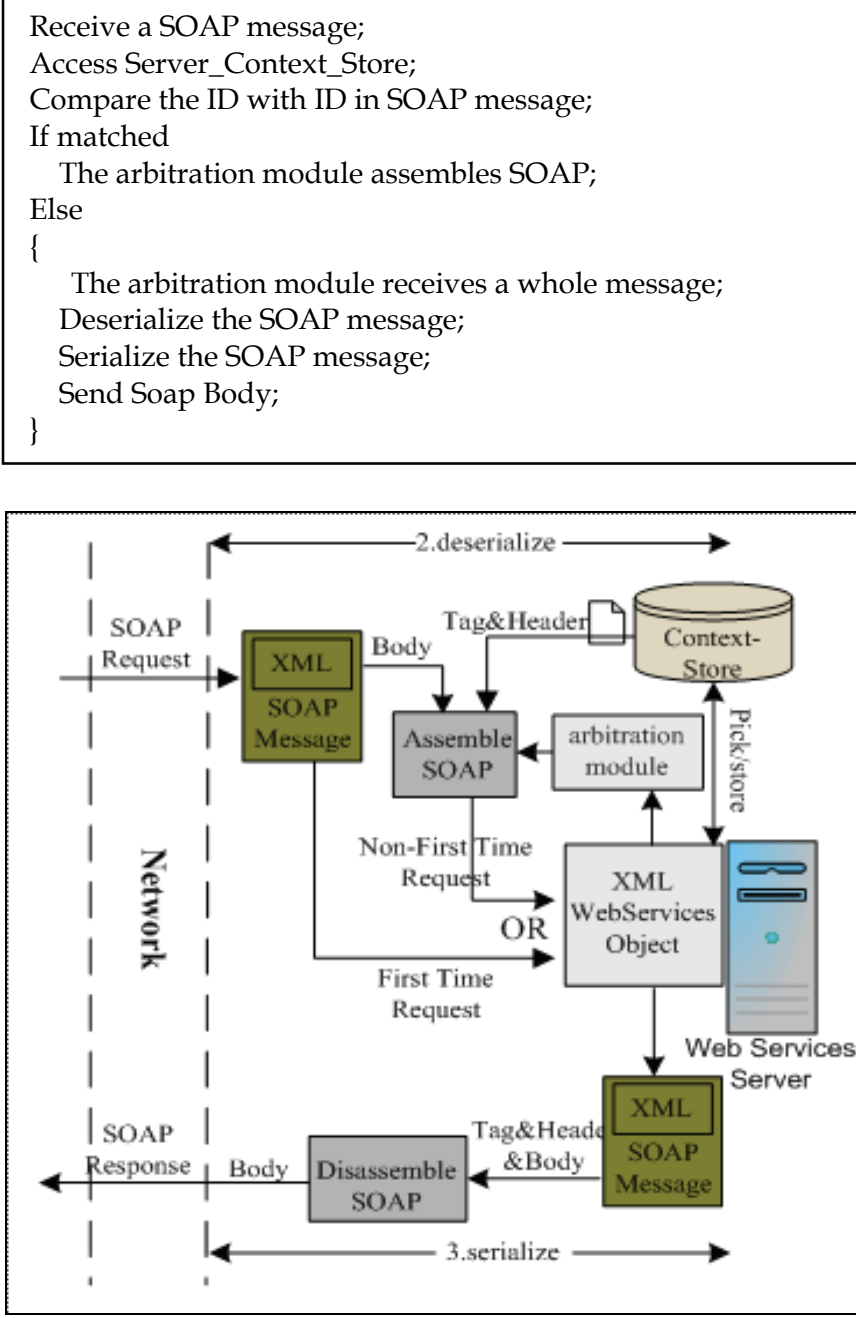

Fig. 6. Algorithm on Web Services server

\section{Experiments}

We construct the following environment to test our approach. The mobile client side adopts PDA, which installs J2ME and the Wireless Toolkit 2.5.2 development tool. The server side installs the Intel Pentium $4 \mathrm{CPU}, 3.0 \mathrm{GHz}, 768 \mathrm{MByte}$ RAM, Bandwidth 100Mbps, and Microsoft Server 2003 operating system, and Dot Net SOAP Engine expansion system, IIS 
6.0. Under such configuration, the result of our experiment is shown in Fig.7. The result in an Internet mobile computing environment is indicated in Fig.8.

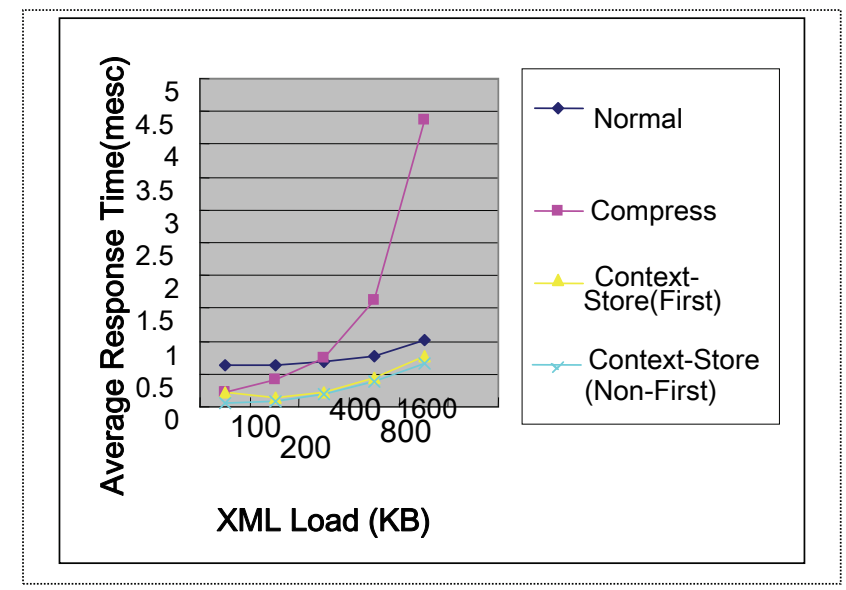

Fig. 7. Comparisons under Wireless LAN

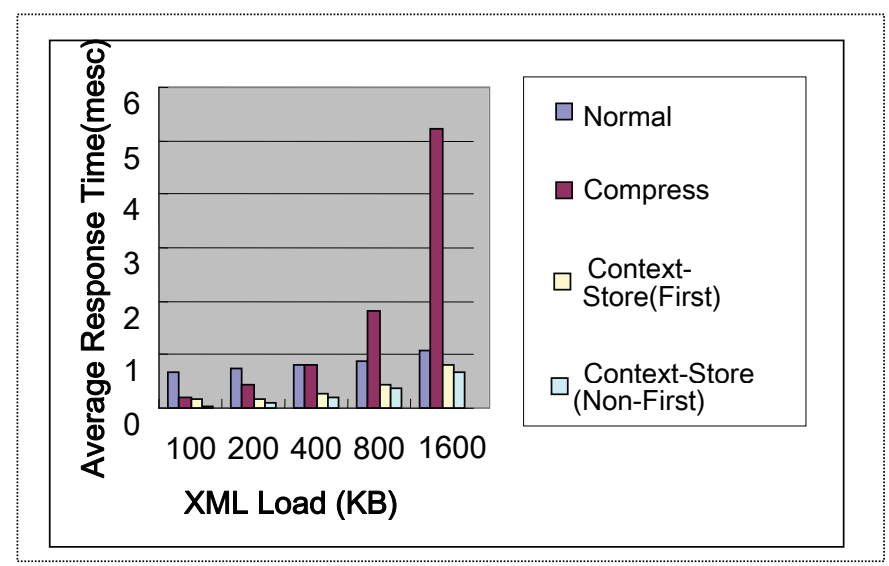

Fig. 8. Comparisons under mobile computing environment 
We packed several SOAP messages in the length of 100, 200, 400, 800, 1600 Kilo Bytes with the contents of Production ID, material, tested date and so on. Under the methods of normal SOAP transmission, Compressing and our Context-Store transmission, all the average response time increases as the length of the SOAP message augments. But apparently, our Context-Store approach has the least time cost compared with the other 2 methods. For the Compressing method, due to the plenty of time consumed by CPU and the limited processing ability, the performance becomes worse even though it cost less time in data transmission. For the Context-Store approach, owing to the only dynamic part of SOAP message transmission, the average response time reduces. In other words, our approach greatly enhances the performance of SOAP message transmission efficiency.

\section{Application}

Based on the above design and experiments, the prototype system has been implemented and applied to production lines in a manufactory, shown in Fig. 9. In the manufactory,

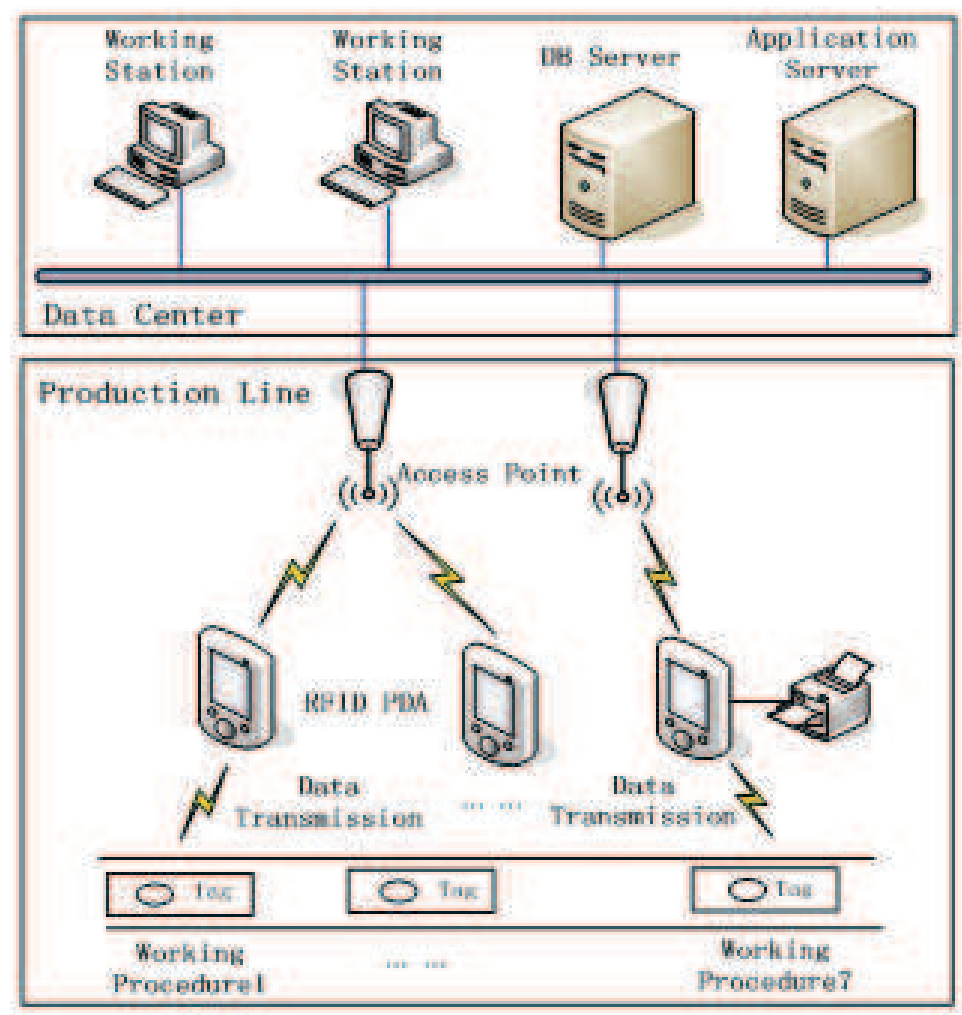

Fig. 9. Application example 
considering the noisy surroundings, the limitations of temperature and humidity and the difficulty of network cabling, we choose PDA with RFID embedded communication module as readers rather than wired RFID readers. The PDA access LAN through Access Point (AP) at the frequency of $2.4 \mathrm{GHz}$.While the embedded communication module exchanges data with tags at the frequency of $902 \mathrm{MHz}$ which is also of free Industrial Scientific Medical (ISM) band. So there is no radio frequency interference. Other application softwares are deployed as follows: working station installs Microsoft Server 2003 operating system, Message Queue Component and Dot Net Framework2.0. The application server installs Internet Information Services 6.0 as WEB server.

In such application scene, workers read information of products with PDA in their hands from 1.0 meters away and record important process information into tags. Meanwhile, through WLAN, By virtue of Web Services, the PDA clients access application server to update process data. With our proposed approach, the performance of data exchange is improved and the production efficiency is increased.

\section{Conclusion}

In this paper, we introduce the widely applied RFID middlewares with the technique of Web Services. More importantly, we design and realize a Context-Store approach to improve the performance of data transmission between mobile client and Web Services server. The experiments show our approach greatly reduces the average response time of SOAP messages, thus achieve its original goal. What's more, the application example of the production lines in manufactory indicates an increase of the production efficiency. However, the symmetrical Context-Store in both mobile client and Web Service server may lead to data inconsistency. Our future work will focus on exploring a more effective storage structure or seek for a way of keeping data consistency.

\section{Acknowledgments}

This research was supported by "Ambient SoC Global COE Program of Waseda University" from the Ministry of Education, Culture, Sports, Science and Technology of Japan and CREST of JST, Japan.

\section{References}

B S Prabhu; Xiaoyong Su \& Harish Ramamurthy. (2006). WinRFID:A Middleware for the Enablement of Radio Frequency Identification (RFID) Based Applications. In: Mobile, Wireless and Sensor Networks: Technology, Applications and Future Directions . John Wiley, 2006.

Christian Floerkemeier \& Matthias Lampe.(2005). RFID middleware design: addressing application requirements and RFID constraints, Proceedings of 2005 joint conference on Smart objects and ambient intelligence: innovative context-aware services: usages and technologies, page :219-224, ISBN:1-59593-304-2, Grenoble, France, 2005, ACM New York. 
Clemens Kerer; Schahram Dustdar \& Mehdi Jazayeri.(2004). Presence Aware Infrastructure Using Web Services and RFID Technologies. Proceedings of the ECOOP Workshop, 2004.

Han Chen; Paul B. Chou; Sastry Duri; Jeffery G. Elliott \& Johnathan M. Reason. (2005). A Model-Driven Approach to RFID Application Programming and Infrastructure Management, Proceedings of IEEE International Conference on e-Business Engineering .

Julio Fernandez;Ana Fernandez \& Jose Pazos. (2005). Optimizing web services performance using caching, Proceedings of the International Conference on Next Generation Web Services Practices, 6 pages, 2005.

K Devaram \& D Andresen. (2003). SOAP Optimization via Parameterized Client-Side Caching, Proceedings of the IASTED International Conference on Parallel and Destributed Computing and Systems, page : 785-790. Marina Del Reg, CA, 2003.

Mike Nikitas. (2003). Improve XML Web Services' Performance by Compressing SOAP. URI: http://www.dotnetjunkies.com/Article/466 30 AE 2-1C79-4D5F-827E6C2857FF1D23.dcik, 2003.

Niranjan \& Aura Ganz. (2004). REALMS-RFID Enabled Animated Space. Proceedings of International Conference on Communication and Computer Network, MIT, MA.

Noah Mendelsohn; Mark Nottingham \& Hervé Ruellan.(2004). XML-binary Optimized Packaging W3C Working Draft. URI: http://www.w3.org/TR/2004/WD-xop1020040608/, 2004, 06.

Seshasayee B; Schwan K\& Widener P. (2004). SOAP-binQ: High-Performance SOAP with Continuous Quality Management, Proceedings of the 24th International Conference on Destributed Computing System, page : 158-165.

Taesu Cheong \& Youngil Kim. (2005). RFID Data Management and RFID Information Value Chain Support with RFID Middleware Platform Implementation , In: On the Move to Meaningful Internet Systems 2005: CoopIS, DOA, and ODBASE, Editors: Agia Napa, Cyprus, page : 557-575, Publisher: Springer Berlin, ISBN : 978-3-540-29736-9, Heidelberg Germany .

Toyotaro Suzumura;Toshiro Takase \& Michiaki Tatsubori. (2005). Optimizing web services performance by differential deserialization, Proceedings of the IEEE International Conference on Web Services. page:185-192,2005.

V Prabakar; Dr. BV Kumar \& SV Subrahmanya. (2006). Management of RFID-centric business networks using Web Services, Proceedings of the Advanced International Conference on Telecommunications and International Conference on Internet and Web Applications and Services, page 133.

World Wide Web Consortium. (2007). SOAP Version 1.2 Part 1: Messaging Framework (Second Edition), W3C Recommendation 27 April 2007. http://www.w3.org/TR/soap12-part1/ . Editors: Martin Gudgin, Marc Hadley \& Noah Mendelsohn etc.

World Wide Web Consortium. (2008). Extensible Markup Language (XML) 1.0 (Fifth Edition) W3C Recommendation 26 November 2008. http://www.w3.org/TR/RECxml/ Editors:Tim Bray, Jean Paoli \& C. M. Sperberg-McQueen etc. 
Young-II Kim; Joo-Sang Park \&Tae-Su Cheong. (2005).Study of RFID middleware framework for ubiquitous computing environment, Proceedings of 7 th International Conference on Advanced Communication Technology, page: 825-830, DOI: 10.1109/ICACT.2005.246078, Korea,July,2005, Seoul, Korea. 


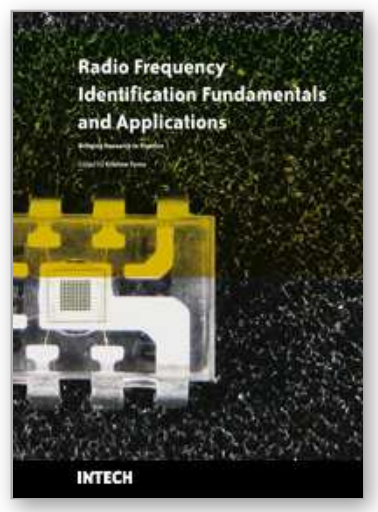

\author{
Radio Frequency Identification Fundamentals and Applications \\ Bringing Research to Practice \\ Edited by Cristina Turcu
}

ISBN 978-953-7619-73-2

Hard cover, 278 pages

Publisher InTech

Published online 01, February, 2010

Published in print edition February, 2010

The number of different applications for RFID systems is increasing each year and various research directions have been developed to improve the performance of these systems. With this book InTech continues a series of publications dedicated to the latest research results in the RFID field, supporting the further development of RFID. One of the best ways of documenting within the domain of RFID technology is to analyze and learn from those who have trodden the RFID path. This book is a very rich collection of articles written by researchers, teachers, engineers, and professionals with a strong background in the RFID area.

\title{
How to reference
}

In order to correctly reference this scholarly work, feel free to copy and paste the following:

Hongying Liu, Satoshi Goto and Junhuai Li (2010). An Efficient Approach for Data Transmission in RFID Middleware, Radio Frequency Identification Fundamentals and Applications Bringing Research to Practice, Cristina Turcu (Ed.), ISBN: 978-953-7619-73-2, InTech, Available from:

http://www.intechopen.com/books/radio-frequency-identification-fundamentals-and-applications-bringingresearch-to-practice/an-efficient-approach-for-data-transmission-in-rfid-middleware

\section{INTECH}

open science | open minds

\section{InTech Europe}

University Campus STeP Ri

Slavka Krautzeka 83/A

51000 Rijeka, Croatia

Phone: +385 (51) 770447

Fax: +385 (51) 686166

www.intechopen.com

\section{InTech China}

Unit 405, Office Block, Hotel Equatorial Shanghai

No.65, Yan An Road (West), Shanghai, 200040, China

中国上海市延安西路65号上海国际贵都大饭店办公楼 405 单元

Phone: +86-21-62489820

Fax: $+86-21-62489821$ 
(C) 2010 The Author(s). Licensee IntechOpen. This chapter is distributed under the terms of the Creative Commons Attribution-NonCommercialShareAlike-3.0 License, which permits use, distribution and reproduction for non-commercial purposes, provided the original is properly cited and derivative works building on this content are distributed under the same license. 